\title{
Refino de óleo de farelo de arroz (Oryza sativa L.) em condições brandas para preservação do $\gamma$-orizanol
}

Refining of rice bran oil (Oryza sativa L.) to preserve $\gamma$-orizanol

\author{
Luz Maria PAUCAR-MENACHOㄹ, Leomar Hackbart da SILVA ${ }^{1}$, Anderson de Souza SANT’ANA², \\ Lireny Aparecida Guaraldo GONÇALVES ${ }^{1 *}$
}

\begin{abstract}
Resumo
O $\gamma$-orizanol é um antioxidante presente no óleo de farelo de arroz, mas ausente em outros óleos vegetais, ao qual têm sido atribuídos efeitos antioxidante e hipocolesterolêmico. O conteúdo de $\gamma$-orizanol no óleo de farelo de arroz bruto varia entre $1-2 \%$, mas durante a etapa de neutralização do refino químico, este composto fica retido na borra. O presente trabalho teve por objetivo propor uma técnica alternativa de refino físico em condições brandas para o óleo de farelo de arroz com a finalidade de preservar o $\gamma$-orizanol. Utilizou-se farelo de arroz inativado e extrusado obtido a partir do processo de produção do arroz parboilizado, sendo o óleo bruto (Oryza sativa) extraído pelo método de expeller. O processo de refino do óleo bruto consistiu nas etapas de degomagem ácida (com $\mathrm{H}_{3} \mathrm{PO}_{4} 85 \%$ ), centrifugação, clarificação, desodorização a $180{ }^{\circ} \mathrm{C}$ e winterização. Foram realizadas análises para caracterização e comparação do óleo bruto e refinado, seguindo metodologias oficiais da AOCS. Concluiu-se que o processo de refino físico preservou $97 \%$ do $\gamma$-orizanol, o qual potencializou a estabilidade oxidativa do óleo refinado pelo método alternativo, que apresentou uma melhoria de $33 \%\left(13,3\right.$ horas a $\left.110{ }^{\circ} \mathrm{C}\right)$ do período de indução da oxidação em relação ao óleo comercial.

Palavras-chave: Óleo de farelo de arroz; refino físico; $\gamma$-orizanol.
\end{abstract}

\begin{abstract}
$\gamma$-orizanol is an antioxidant present in rice bran oil, but absent in other vegetable oils, which were attributed to antioxidant and hypocholesterolemic effects. The content of $\gamma$-orizanol in crude rice bran oil varies between $1-2 \%$, but during neutralization in chemical refining this substance is transferred to soapstock. The aim of this research is to study an alternative technique of physical refining in light conditions with the purpose of preserving $\gamma$-orizanol. Inativacted and extrused rice bran obtained by the production of parboilizated rice was used to extract crude rice bran oil (Oryza sativa) by the expeller method. The process of refining consisted of: acid degumming (with $85 \% \mathrm{H}_{3} \mathrm{PO}_{4}$ ), centrifugation, clarification, deodorization, and winterization. Chemical analyses were carried out by the characterization of refined oil according to the AOCS methods. Results showed that the physical refining process was able to preserve $97 \%$ of $\gamma$-orizanol, and its presence improved the oxidative stability of the oil comparing with the commercial rice bran oil (by $33 \% 13.3$ hours to $110^{\circ} \mathrm{C}$ ).

Keywords: Rice bran oil; physical refining; $\gamma$-oryzanol.
\end{abstract}

\section{Introdução}

O comércio global de arroz se expandiu a uma taxa média de $7 \%$ ao ano na década de 90 , alcançando aproximadamente 25 milhões de toneladas no final desse período. China e Índia respondem juntas por mais da metade da produção mundial. O Brasil foi o $9^{\circ}$ maior produtor deste cereal em 2005, atingindo uma produção de mais de 13 milhões de toneladas métricas ${ }^{14}$.

O grão de arroz é composto por aproximadamente 20\% de casca, $70 \%$ de endosperma e $10 \%$ de farelo e germe. Nas camadas do farelo e germe, está concentrada a maior parte dos lipídeos do grão de arroz ${ }^{29}$. Durante o descascamento, obtémse o farelo (pericarpo e gérmen) que representa de 5,0 a 5,5\% do grão inteiro. O farelo contém entre $12-18 \%$ de óleo, o que significa que o grão de arroz potencialmente possui apenas $0,8 \%$ de óleo ${ }^{11}$ e é por este motivo que a extração do óleo é feita a partir do farelo de $\operatorname{arroz}^{25,43}$.

\footnotetext{
Departamento de Tecnologia de Alimentos,

Faculdade de Engenharia de Alimentos - FEA

Universidade Estadual de Campinas - UNICAMP, Rua Monteiro Lobato, 80

CP 6091, CEP 13081970, Campinas - SP, Brasil

E-mail:lireny@fea.unicamp.br).

Departamento de Ciência de Alimentos,

Faculdade de Engenharia de Alimentos - FEA

Universidade Estadual de Campinas - UNICAMP, Campinas - SP, Brasil

*A quem a correspondência deve ser enviada
}

Durante o beneficiamento do arroz várias partes dos grãos são removidas e há a concentração dos principais elementos nutritivos no farelo, o que o torna ótima matéria-prima para processos industriais e para alimentação, mas sua utilização está limitada pela atividade enzimática que se desenvolve a partir do beneficiamento. O farelo de arroz contém vários sistemas enzimáticos, dentre os quais se destaca a presença de enzimas $\alpha$-amilase, $\beta$-amilase, catalase, sendo as mais importantes as lipases, lipoxigenases e peroxidases ${ }^{25}$. As enzimas lipolíticas, as quais são ativadas na etapa de polimento do grão de arroz, hidrolisam os triglicerídeos do produto e aumentam rapidamente a quantidade de ácidos graxos livres, reduzindo o rendimento em óleo neutro e dificultando seu refino $^{18}$. Essas reações desencadeiam processos de rancidez oxidativa e hidrolítica que devem ser controlados para evitar a degradação do farelo de arroz e permitir sua utilização como matéria-prima para obtenção de óleo comestível. Isto pode ser conseguido pela estabilização do farelo por métodos físicos ou químicos ${ }^{33}$ ou pela extração do óleo imediatamente após a obtenção do farelo ${ }^{18}$.

Entre 90-96\% dos lipídeos componentes do óleo de farelo bruto de arroz são materiais saponificáveis (triacilgliceróis, diacilgliceróis, monoacilgliceróis, ácidos graxos livres e ceras) e 3-5\% são insaponificáveis (esteróis, tocoferóis, tocotrienóis, álcoois triterpênicos) ${ }^{29}$. Dentre os ácidos graxos, destacam-se 
o palmítico (21-26\%), linoléico (31-33\%) e oléico (37-42\%) ${ }^{21}$, e, devido ao seu alto conteúdo de ácidos graxos monoinsaturados e poliinsaturados, é considerado um óleo saudável ${ }^{39}$. O conteúdo de matéria insaponificável varia dependendo do grau e método de processamento, todavia o óleo bruto de farelo de arroz possui um teor ao redor de $4 \%$ quando comparado a outros óleos vegetais que apresentam um teor médio de $1 \%{ }^{26}$.

Os benefícios associados ao óleo de farelo de arroz devemse não somente à sua composição triacilglicerídica adequada, mas, principalmente, à fração insaponificável do óleo ${ }^{27,29}$. Estes componentes são também importantes na prevenção e terapia associados a problemas no metabolismo de gorduras ${ }^{12}$. Além de altos níveis de tocoferóis, tocotrienóis e fitosteróis que conferem resistência à oxidação e deterioração, o óleo de farelo de arroz tem sua estabilidade aumentada devido à presença de um antioxidante ausente em outros óleos, o $\gamma$-orizanol ${ }^{34}$, ao qual, tem sido atribuído efeito também hipocolesterolêmico ${ }^{20,36} \cdot \gamma$-orizanol é constituído de uma mistura de ésteres de ácido ferúlico, sendo os mais importantes ciclo artenil ferulato, 24-metileno cicloartenil ferulato, $\beta$-sitosteril ferulato e campesteril ferulato. Na Figura 1, é mostrada a estrutura química do 24-metileno cicloartenil ferulato, o componente principal do $\gamma$-orizanol ${ }^{30}$.

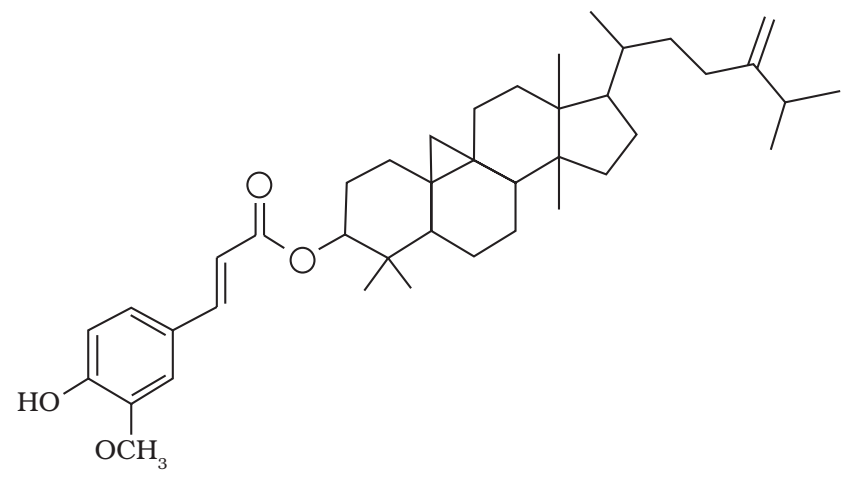

Figura 1. Estrutura do 24-metileno cicloartenil ferulato.

O efeito antioxidante dos ferulatos pode ser explicado similarmente ao dos antioxidantes sintéticos, pela formação de diversas estruturas estabilizadas por ressonância ${ }^{42}$.

JULIANO et al. ${ }^{22}$, avaliaram a capacidade antioxidante do $\gamma$-orizanol, seu mecanismo de ação e efeitos sobre a estabilidade oxidativa de óleos farmacêuticos. Os resultados obtidos indicam que o $\gamma$-orizanol pode ter aplicação potencial como antioxidante natural para a estabilização oxidativa de óleos. Suas propriedades antioxidantes podem ainda ser melhoradas através da sua associação com outros antioxidantes naturais, obtendo-se misturas capazes de substituir os antioxidantes sintéticos. O fato de possuir outras atividades biológicas (anticolesterolêmico, proteção solar, etc.), o torna um componente multifuncional para formulações farmacêuticas, cosméticas e alimentícias. $\mathrm{O}$ óleo de arroz, fonte rica em $\gamma$-orizanol e tocoferóis, torna-se bastante atrativo para novas aplicações tópicas. A importância de se manterem os níveis de $\gamma$-orizanol naturalmente presentes no óleo de farelo de arroz pode ser justificada pelo estudo realizado por BERGER et al. ${ }^{5}$, que avaliaram as propriedades hipocolesterolêmicas do óleo de arroz, com diferentes teores de $\gamma$-orizanol, em homens hipercolesterolêmicos. Verificou-se que o óleo de arroz foi efetivo na redução do colesterol total (11,9\% de redução, após duas semanas de ingestão do óleo) e na redução da relação do LDL-C/HDL-C, atingindo valores de redução de 19\% em quatro semanas, indicando que o óleo de arroz pode ser benéfico na manutenção de níveis adequados de colesterol no plasma.

O conteúdo de $\gamma$-orizanol no óleo de farelo de arroz bruto varia entre $1,5-2,9 \%^{17,28,34,41,44}$. Diferenças no conteúdo de $\gamma$-orizanol podem ser justificadas pela variedade e tipo de processamento dos grãos de arroz, perdas no processamento em virtude do tipo de extração e refino do óleo, além dos parâmetros empregados no seu processamento ${ }^{34}$. Normalmente o óleo de arroz requer refino químico que adiciona soda cáustica no óleo na etapa de neutralização. O óleo após ser neutralizado exige duas lavagens sucessivas com água branda, secagem sob vácuo para eliminação da umidade residual e uma etapa de desceragem antes da desodorização ${ }^{30}$. No entanto, durante a etapa de neutralização do refino químico, o $\gamma$-orizanol é quase totalmente transferido para a borra (soapstock), um subproduto desta etapa do processo ${ }^{29}$.

O conteúdo de $\gamma$-orizanol no óleo de farelo de arroz depende do método de refino usado. No refino químico, podem-se ter perdas ao redor de $90 \%$ deste composto ${ }^{17}$. ORTHOEFER ${ }^{29}$ verificou redução do conteúdo de $\gamma$-orizanol de 2,0 para $0,1 \%$ ao usar o refino químico e redução para a faixa de 1,0-1,5\%, ao se usar o refino físico. O refino físico contempla uma etapa de superdegomagem com ácido fosfórico, clarificação e desodorização. O teor de $\gamma$-orizanol encontrado nos óleos refinados de farelo de arroz e comercializados por diferentes processadores varia entre 0,0144 e $0,0787 \%^{31}$. No refino químico é usado álcali para desacidificação, enquanto que no refino físico esta desacidificação é realizada por destilação. As etapas do processo de refino químico constituem-se basicamente por: degomagem, neutralização, branqueamento, desceragem e desodorização ${ }^{25,35}$. Na etapa de neutralização, ocorre a conversão dos ácidos graxos livres em sabões através da reação com hidróxido de sódio, os quais devem ser separados por centrifugação, dando origem à borra. Devido ao seu alto teor de ácidos graxos livres, esta é a etapa de maior dificuldade no processamento de óleo de farelo de $\operatorname{arroz}^{23}$, já que a formação da borra leva à maior perda do $\gamma$-orizanol. $O$ teor de $\gamma$-orizanol na borra pode oscilar de 1,19 a 3,6\% em base seca ${ }^{17,35,37}$.

Além da desvantagem da perda do $\gamma$-orizanol para a borra no caso do refino químico, o refino do óleo de farelo de arroz é considerado bastante difícil devido ao alto conteúdo de ácidos graxos livres, ceras e pigmentos ${ }^{10,15}$. Assim, algumas técnicas para refino têm sido desenvolvidas, como o refino em miscela ${ }^{6}$, o refino em mistura de solventes (GOENKA, 1983 apud BHATTACHARYA et $\mathrm{al}^{7}$ ) e a reesterificação dos ácidos graxos livres com monoacilgliceróis ${ }^{9}$. Apesar de algumas técnicas conseguirem reduzir as perdas de $\gamma$-orizanol, estas requerem um custo inicial para montagem ou adaptação das linhas de processamento, além das desvantagens adicionais como o uso de solventes que podem ser inflamáveis e provocar riscos ao meio ambiente. 
Desta forma, o presente trabalho teve por objetivo estudar uma técnica alternativa de refino físico em condições brandas aplicada ao óleo de farelo de arroz obtido pelo método de expeller com a finalidade de preservar o $\gamma$-orizanol, evitandose a etapa de neutralização química.

\section{Material e métodos}

\subsection{Material}

O óleo bruto de farelo de arroz foi obtido a partir do arroz parboilizado. A inativação do farelo de arroz ocorre no próprio processo de parboilizacão, no qual os grãos, após o encharcamento em água a $60{ }^{\circ} \mathrm{C}$ por aproximadamente 6 horas, são submetidos à autoclavagem por 10 a 30 minutos, sob pressão de 0,5 a 1,0 Kgf.cm ${ }^{-2}$, promovendo a inativação das lipases e lipoxigenases ${ }^{38}$.

O farelo de arroz inativado no processo de parboilização foi submetido ao método de expeller para a obtenção do óleo bruto, sendo gentilmente cedido pela Indústria de Beneficiamento de Arroz Parboilizado - Urbano Agroindústrias Ltda. (Unidade Jaraguá do Sul - SC). A tecnologia de extrusão foi adotada como alternativa para a extração de óleo em equipamento industrial, obtendo-se um lote de óleo bruto que ficou estocado por $7 \mathrm{me}$ ses para verificar sua viabilidade de refino.

\subsection{Métodos}

\section{Métodos de avaliação da qualidade da matéria-prima}

As metodologias e condições utilizadas foram as seguintes:

- Percentagem de Ácidos Graxos Livres: método Ca 5a-40, AOCS $^{3}$, sendo $\mathrm{f}=1,99$ utilizado para cálculo de índice de acidez (mg de KOH.g ${ }^{-1}$ );

- Umidade e Matéria Volátil: método Ca 2c-25, $\mathrm{AOCS}^{3}$;

- Índice de peróxido: método $\mathrm{Cd} 8 \mathrm{~b}-90, \mathrm{AOCS}^{3}$;

- Teor de fósforo: método DGF CIII-16a (1989), com medições usando o espectrofotômetro UV/VIS Lambda 20 - Perkin Elmer, em comprimento de onda de $830 \mathrm{~nm}$, de acordo com ESTEVES, GONÇALVES e BARRERAARELLANO $^{13}$. Amostras de óleo que sofreram adição de ácido fosfórico para degomagem foram previamente lavadas com água para remoção do fósforo inorgânico a fim de quantificar apenas o fósforo orgânico dos fosfolipídeos;

- Cor Lovibond: Método Cc 13e-92 - AOCS $^{3}$, utilizando-se o Lovibond Tintometer model E e cubetas de $5^{1 / 4^{\prime \prime}}$ e

- Estabilidade oxidativa: método Cd 12-b92, utilizando-se o Oxidative Stability Instrument (OSI) Omniom Archer - Daniels Midland Company - AOCS ${ }^{3}$, com 5 g de amostra, fluxo de 9,0 L.h ${ }^{-1}$ e temperatura $=110^{\circ} \mathrm{C}$.

\section{Métodos de identificação da matéria-prima}

- Densidade: medição por densímetro marca Precision, calibrado a $20{ }^{\circ} \mathrm{C}$ (faixa de medição 0,900-0,950);
- Índice de refração: método Cc-7-25, $\mathrm{AOCS}^{3}$, utilizando refratômetro ABBÉ acoplado a banho Lauda M3;

- Índice de iodo: método Cd-1d-92, $\mathrm{AOCS}^{3}$, calculado a partir da composição em ácidos graxos;

- Índice de saponificação: método Cd 1c-85, $\mathrm{AOCS}^{3}$, calculado a partir da composição em ácidos graxos;

- Composição em ácidos graxos: a etapa de esterificação foi realizada segundo HARTMAN e LAGO ${ }^{19}$ e a de composição em ésteres metílicos dos ácidos graxos, segundo $\mathrm{AOCS}^{3}$, método Ce 1-62, utilizando cromatógrafo gasoso capilar - CGC AGILENT 6850 SERIES GC SYSTEM, coluna capilar: DB-23 AGILENT (50\%cyanopropyl - methylpolysiloxane), dimensões $60 \mathrm{~m}$, diâmetro interno 0,25 mm, espessura do filtro $0,25 \mu \mathrm{m}$. Condições de operação do cromatógrafo: temperatura do detector $=280{ }^{\circ} \mathrm{C}$; temperatura do injetor $=250{ }^{\circ} \mathrm{C}$; temperatura do forno $=$ $110{ }^{\circ} \mathrm{C}-5$ minutos, $110^{\circ} \mathrm{C}-215^{\circ} \mathrm{C}\left(5^{\circ} \mathrm{C} / \mathrm{min}\right), 215^{\circ} \mathrm{C}$ por 24 minutos; Gás de arraste $=$ hélio; volume injetado $=$ 1,0 $\mu \mathrm{L}$; Split: 1:50; e

- Teor de $\gamma$-orizanol: método segundo ROGERS, et al. ${ }^{31}$, com metodologia modificada por SCAVARIELLO ${ }^{34}$, em cromatógrafo líquido de alta eficiência (HPLC) - Perkin Elmer 10 em condições isocráticas. Detector UV/Visível: comprimento de onda $315 \mathrm{~nm}$. Coluna: Hypersil ODS

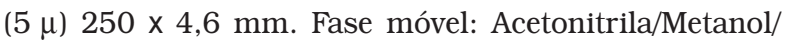
Isopropanol 50/45/5 (v/v/v). Fluxo da Fase móvel: 1,0 mL. min $^{-1}$. Volume injetado: $20 \mu \mathrm{L}$. Padrão secundário de $\gamma$-orizanol, lote F02627, fornecido pela empresa Tsuno Rice Fine Chemicals. Pureza - 99,3\%. Quantificação através de curva de calibração externa. A identificação dos picos do $\gamma$-orizanol foi realizada através da comparação dos tempos de retenção efetivada nas mesmas condições, considerando os picos predominantes do $\gamma$-orizanol reportados por Rogers et al. ${ }^{31}$, que utilizou a identificação por CG/MS do mesmo padrão fornecido pela Tsuno Rice Chemicals.

\section{Refino do óleo de farelo de arroz}

As unidades utilizadas na planta piloto do laboratório de óleos e gorduras-FEA-UNICAMP para degomagem e clarificação consistiram em sistemas de reatores de vidro encamisados com capacidade de 2 litros, dotados de sistema de agitação pneumática acopladas a sistema de aquecimento com banho Lauda C20. A centrífuga utilizada consistiu no modelo MTD III Plus.

O óleo bruto foi primeiramente analisado quanto ao índice de acidez, teor de fósforo e cor.

O processo de refino do óleo bruto de farelo de arroz consistiu das etapas de degomagem ácida (com ácido fosfórico 85\%), centrifugação, clarificação, nova centrifugação, desodorização, winterização e filtragem final (Figura 2).

A degomagem foi realizada em lote de 2 litros pela adição de $0,3-0,5 \%$ em peso de ácido fosfórico $85 \%$, ao óleo bruto previamente aquecido a $80{ }^{\circ} \mathrm{C}$, sendo em seguida agitado por 
10 minutos no reator. Decorrido este tempo, foi adicionado $1 \%$ de água, sendo o óleo mantido sob agitação por mais $10 \mathrm{minu-}$ tos. As amostras de óleo foram então centrifugadas a $3.300 \mathrm{rpm}$ por 5 minutos para eliminação das lecitinas.

A clarificação consistiu na adição de $2 \%$ em peso de terra clarificante Tonsil actisil - 280FF (Sud Chemie do Brasil Ltda) ao óleo de farelo de arroz e manutenção sob vácuo a uma tem-

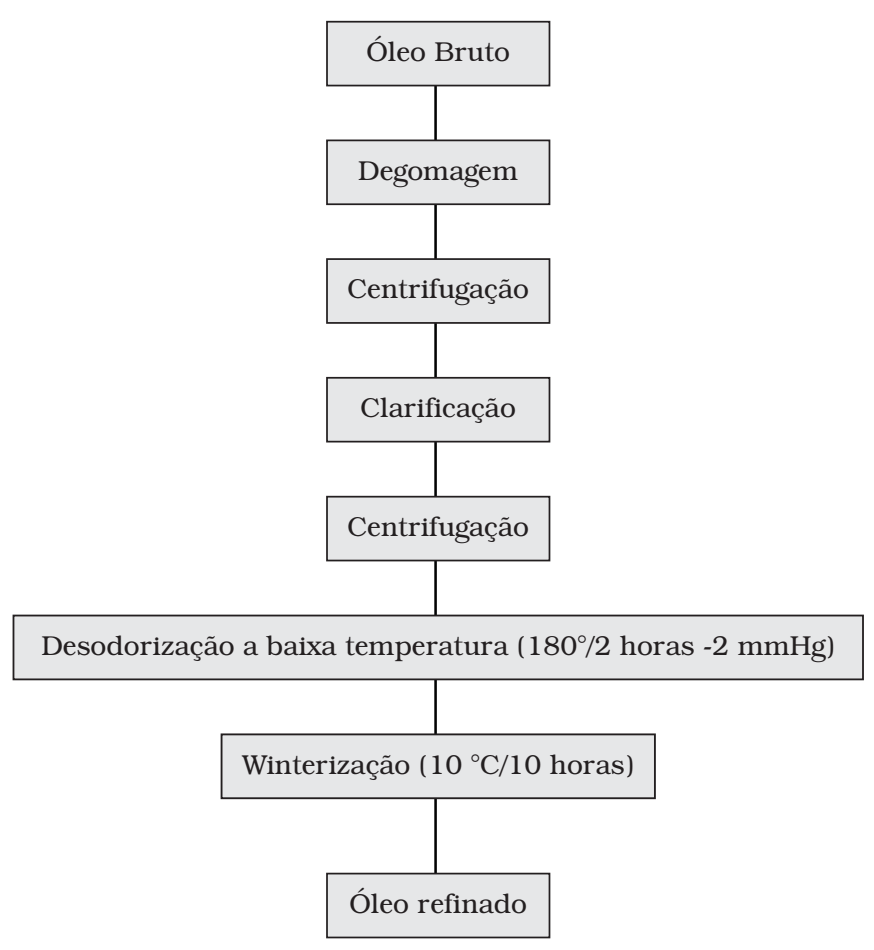

Figura 2. Fluxograma do processo de refino físico do óleo de farelo de arroz em condições brandas. peratura de $90{ }^{\circ} \mathrm{C}$ por 20 minutos com agitação no reator. O óleo foi centrifugado a $3.300 \mathrm{rpm}$ por 5 minutos. As amostras de óleo foram novamente centrifugadas para eliminação da terra clarificante e determinou-se o teor de fósforo.

A desodorização foi realizada a $180{ }^{\circ} \mathrm{C}$ aplicada por um sistema encamisado com óleo de silicone, durante 2 horas sob vácuo de $12 \mathrm{mmHg}$. Ao invés de arraste de vapor recorreu-se ao gás inerte de nitrogênio e à coleta de voláteis do desodorizado através de um banho Lauda K6 a baixa temperatura.

A winterização foi realizada com auxílio de banho termostático Lauda RC6, acoplado a um programador de temperatura PM51 com sistema de agitação IKA, sob as seguintes condições: agitação de $90 \mathrm{rpm}$; redução da temperatura de $30^{\circ} \mathrm{C}$ até $10^{\circ} \mathrm{C}$, a uma taxa de $2{ }^{\circ} \mathrm{C} / \mathrm{h}$. Após atingir-se $10{ }^{\circ} \mathrm{C}$, o óleo foi mantido nesta temperatura, sob agitação, por 4 horas.

Em seguida o óleo winterizado foi filtrado a vácuo em kitassato/funil de Büchner, com auxílio de papel de filtro, Marca White Ribbon.

\section{Resultados e discussão}

A Tabela 1 mostra a composição em ácidos graxos dos óleos analisados. Os ácidos palmítico, oléico e linoléico são os principais ácidos graxos presentes no óleo, constituindo mais de $90 \%$ da porção dos triacilgliceróis (Figura 3).

Embora a legislação brasileira vigente junto a ANVISA (Resolução 482/99 ${ }^{1}$ ) tenha sido revogada em 23/09/2005, a legislação atualmente vigente ${ }^{2}$ remete aos dados do Codex Alimentarius - FAO/OMS ${ }^{8}$. Porém o Codex Alimentarius não contempla óleo de arroz, o que leva a um impasse de em qual legislação se apóia o padrão de identidade e qualidade (PIQ) do óleo de arroz. As Tabelas 1 e 2 mostram os dados que estavam contemplados na resolução revogada, dados estes compilados de amostras de óleos brasileiros analisados em laboratórios de vigilância sanitária no País, anteriores a 1999.

Tabela 1. Composição em ácidos graxos do óleo bruto de farelo de arroz, refinado em condições brandas e óleo comercial de farelo de arroz.*

\begin{tabular}{|c|c|c|c|c|}
\hline Ácido graxo & Óleo bruto & $\begin{array}{c}\text { Óleo refinado } \\
\text { (condições brandas) }\end{array}$ & Óleo refinado comercial & $\begin{array}{l}\text { Requisitos específicos } \\
\text { (RDC } 482 / 99 \text { ANVISA) }^{1}\end{array}$ \\
\hline (C14:0) Ácido mirístico & 0,27 & 0,29 & 0,23 & $0,4-1,0$ \\
\hline (C16:0) Ácido palmítico & 19,90 & 20,35 & 19,01 & $12,0-18,0$ \\
\hline (C16:1) Ácido palmitoléico & 0,03 & 0,26 & 0,15 & $0,2-0,4$ \\
\hline (C18:0) Ácido esteárico & 1,87 & 1,34 & 0,80 & $1,0-3,0$ \\
\hline (C18:1 cis) Ácido oléico & 43,14 & 42,47 & 41,08 & $40,0-50,0$ \\
\hline (C18:3 cis) Ácido linolênico & 1,47 & 1,41 & 1,76 & $<1,0$ \\
\hline (C20:0) Ácido araquídico & 0,79 & 0,77 & 0,69 & $<1,0$ \\
\hline (C20:1) Ácido gadoléico & 0,50 & 0,49 & 0,54 & $<1,0$ \\
\hline (C22:0) Ácido behênico & 0,31 & 0,28 & 0,00 & $<1,0$ \\
\hline (C22:1) Ácido erúcico & 0,04 & 0,03 & 0,23 & - \\
\hline (C24:0) Ácido lignocérico & 0,46 & 0,42 & 0,40 & - \\
\hline Ácidos graxos saturados & 23,6 & 23,5 & 21,1 & - \\
\hline Ácidos graxos poliinsaturados & 32,7 & 33,3 & 36,9 & - \\
\hline Índice de iodo calculado & 96 & 96 & 101 & - \\
\hline Índice de saponificação calculado & 191 & 191 & 191 & - \\
\hline
\end{tabular}

\footnotetext{
*Média de duas repetições.
} 
FID 1, (A:|23NOVO10.D)

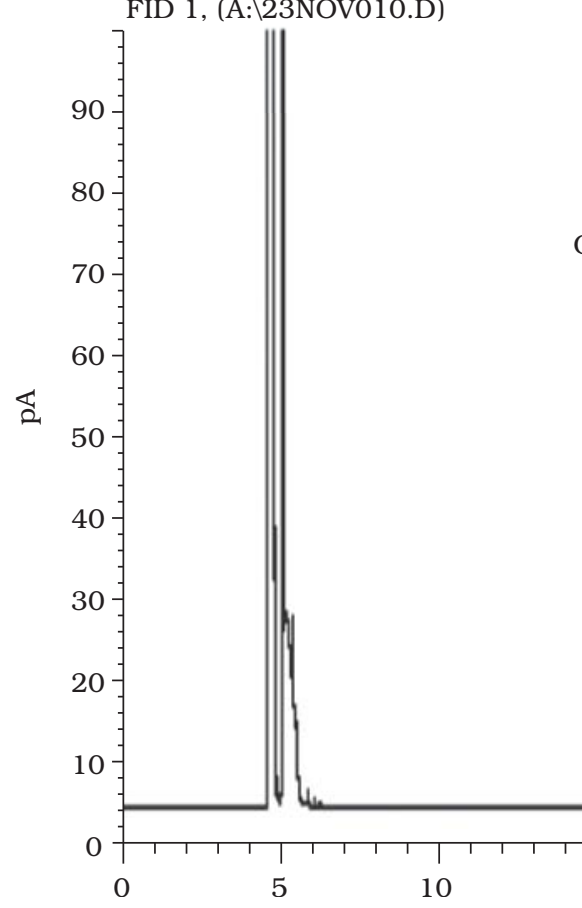

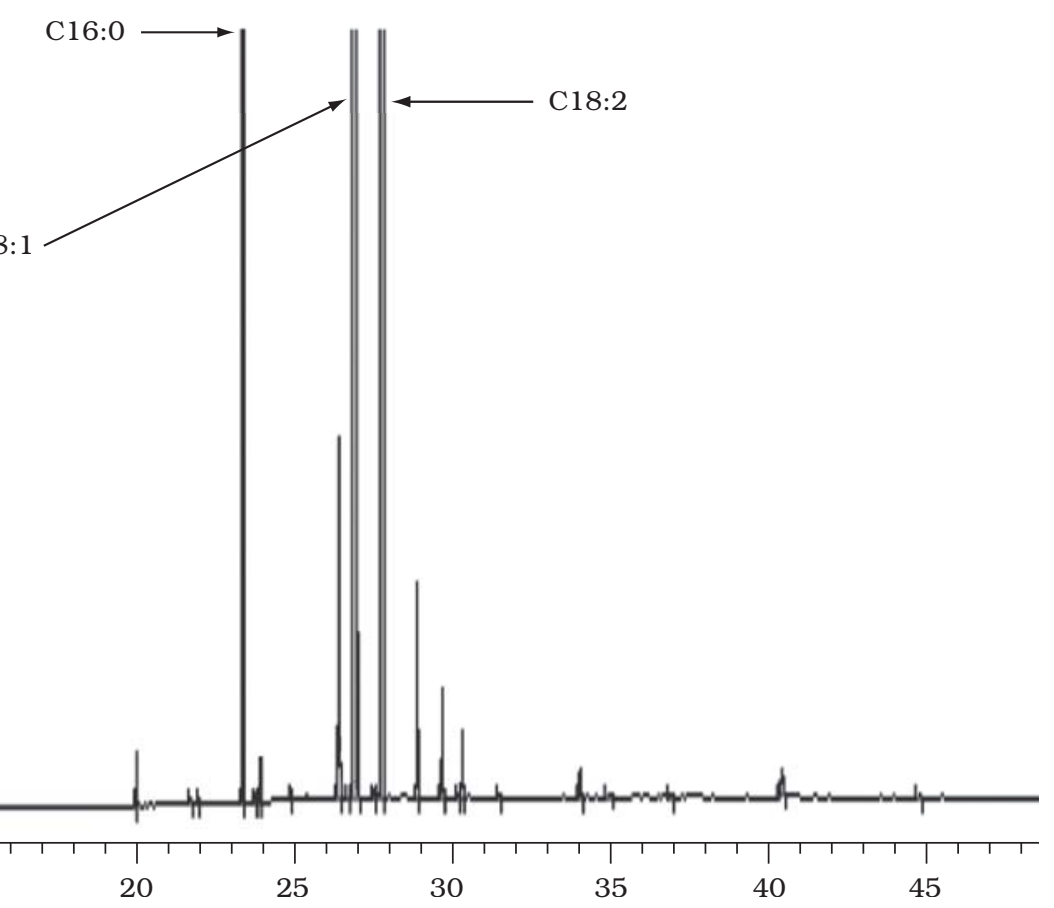

Figura 3. Cromatograma da composição em ácidos graxos do óleo de farelo de arroz. A identificação dos picos cromatográficos está relacionada na Tabela 1.

Tabela 2. Caracterização físico-química do óleo bruto de farelo de arroz e do óleo de farelo de arroz refinado pelo refino físico em condições brandas.

\begin{tabular}{|c|c|c|c|}
\hline Determinações & Óleo bruto & $\begin{array}{c}\text { Óleo refinado } \\
\text { (condições brandas) }\end{array}$ & 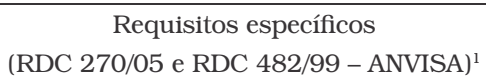 \\
\hline Ácidos graxos livres (\%) & 1,43 & 0,76 & - \\
\hline Índice de acidez (g. $\left.100 \mathrm{~g}^{-1}\right)$ ) & 2,85 & 1,37 & Max. 0,3 \\
\hline Umidade e matéria volátil (\%) & 0,24 & 0,15 & - \\
\hline Densidade $\left(20{ }^{\circ} \mathrm{C} / 20^{\circ} \mathrm{C}\right)$ & 0,921 & 0,915 & $0,916-0,921$ \\
\hline Índice de Refração $\left(\mathrm{n}_{\mathrm{D}}{ }^{40}\right.$ ) & 1,470 & 1,466 & $1,465-1,468$ \\
\hline Índice de iodo & 96 & 96 & 99-108 \\
\hline Índice de Peróxido (meq. $\mathrm{kg}^{-1}$ ) & 17,8 & 7,7 & Max. 10 \\
\hline Teor de fósforo (mg.kg ${ }^{-1}$ ) & 186,5 & 10,4 & - \\
\hline
\end{tabular}

Os resultados da caracterização físico-química do óleo bruto de farelo de arroz e do óleo obtido pelo refino físico e os parâmetros da legislação vigente são apresentados na Tabela 2.

A porcentagem de ácidos graxos livres para o óleo bruto foi próximo de 1,5. Este dado só é encontrado para óleos bem processados e, neste caso, justifica-se pelo fato do farelo ter sido inativado e logo, em seguida, extrusado para ser submetido ao processo de extração por expeller. Esta acidez tão baixa só é possível de ser obtida quando a indústria produtora de arroz tem seu processo de obtenção do óleo dentro da própria planta de produção, permitindo uma inativação eficiente das lipases que atuam fortemente no farelo. Em seu estudo sobre a atividade hidrolítica da lipase durante o armazenamento do farelo de arroz não estabilizado, BELLA CRUZ ${ }^{4}$ observou mudanças na composição dos acilgliceróis nas primeiras 24 horas de armazenamento, ocorrendo uma redução do teor de triacilgliceróis da ordem de $21 \%$ para o farelo armazenado em temperatura ambiente e $23,1 \%$ para o armazenamento em estufa a $30{ }^{\circ} \mathrm{C}$. Em contrapartida, os teores de acilgliceróis parciais e ácidos graxos livres apresentaram acréscimo no mesmo período. Os valores iniciais de 3,8 e 3,3\% aumentaram para 12,9 e 13,7\% de acilgliceróis e ácidos graxos livres, respectivamente, no caso de farelo armazenado à temperatura ambiente. Para $30{ }^{\circ} \mathrm{C}$, foram obtidos os valores de 13,1 e 15,5\%, respectivamente. Óleos brutos de farelo de arroz disponíveis no mercado nacional apresentam teores elevados de acidez na faixa de 10-15\% em ácidos graxos livres ${ }^{16}$ e apresentam colorações extremamente escuras, aproximando-se da cor negra. Quanto maior a porcentagem de ácidos graxos livres no óleo bruto, maiores serão as perdas no refino do óleo ${ }^{24}$. Uma acidez maior que $15 \%$ torna o refino do 
óleo inviável economicamente ${ }^{32}$. Apesar destes valores revelarem uma maior probabilidade à rancidez oxidativa e, portanto, menor estabilidade do óleo durante a estocagem e comercialização, esta tendência não foi observada para o óleo obtido pelo refino físico em condições brandas, devido à manutenção do teor de $\gamma$-orizanol ao redor de $1 \%$ no óleo (Tabela 3 ), o que se comprova pelo período de indução mais longo (13,3 horas) do óleo refinado contra 8,9 horas do óleo comercial (Tabela 4). Desta forma, para o óleo de farelo de arroz, uma acidez mais elevada não levaria a problemas de estabilidade oxidativa, se há a preservação do $\gamma$-orizanol no óleo.

A redução do teor de fósforo de $186,5 \mathrm{mg} \cdot \mathrm{kg}^{-1}$ no óleo bruto para $10,4 \mathrm{mg} \cdot \mathrm{kg}^{-1}$ no óleo refinado pode ser atribuída à etapa de degomagem $(0,3-0,5 \%$ de ácido fosfórico $+1 \%$ de água) e à etapa de clarificação. Nesta última etapa, foi necessário usarse $2 \%$ de terra clarificante para reduzir o teor de fósforo para aproximadamente $10 \mathrm{mg} \cdot \mathrm{kg}^{-1}$. Uma maior quantidade de terra clarificante adicionada poderia tornar este processo inviável economicamente porque as terras clarificantes utilizadas devem ser descartadas em aterros sanitários tornando-se um grave entrave à poluição ambiental, além dos custos envolvidos. Teores de terra entre 0,3-0,5\% são economicamente aceitáveis.

O índice de peróxidos é uma medida do estado de oxidação primária do óleo ou gordura, o qual é influenciado por fatores como ácidos graxos constituintes, tempo e condições de estocagem ${ }^{24}$. No presente estudo, o índice de peróxidos apresentou-se dentro do limite estabelecido pela legislação brasileira (máx. 10 meq. $\mathrm{kg}^{-1}$ ), corroborando a estabilidade oxidativa do óleo obtido pelo refino físico em condições brandas (Tabela 4). Embora o parâmetro de índice de peróxido tenha sido atendido, o óleo aqui avaliado apresentou um grande intervalo de tempo entre o tempo de extração e seu processamento ( 7 meses). Portanto, recomenda-se o refino deste óleo logo após sua obtenção no expeller que contribuirá para que se obtenham valores de índice de peróxido mais baixos que os aqui encontrados. O processamento a baixa temperatura apresentou redução de $43 \%$ nos níveis de oxidação existentes, o que limita até que nível de peróxidos pode-se submeter este óleo ao processo aqui proposto para consumo humano, já que o teor de $\gamma$-orizanol deve ser preservado.

A medida do índice de iodo retrata a presença de sítios ativos de duplas ligações dos ácidos graxos ${ }^{24}$. O índice de iodo do óleo de farelo de arroz é bastante elevado (99-108) ${ }^{40}$, o que atesta sua elevada insaturação (aproximadamente 75\%).

A determinação de $\gamma$-orizanol, por cromatografia em fase líquida de alta pressão em amostra padrão e nos óleos de farelo de arroz comercial, bruto e refinado está apresentada na Tabela 3 e na Figura 4. Os valores estão de acordo com a literatura, não havendo alterações no percentual de orizanol quando se utiliza refino físico ${ }^{29}$. No entanto, quando se utiliza refino químico a perda desse composto é quase total, sendo esse comportamento também observado em experimentos realizados por GOPALA KRISHNA et al. ${ }^{17}$.

Na Tabela 4, estão descritos os períodos de indução, em horas, da oxidação do óleo refinado pelo método físico em condições brandas e do óleo comercial de farelo de arroz. Obser-
Tabela 3. Concentração de $\gamma$-orizanol em óleos de farelo de arroz.

\begin{tabular}{lc}
\hline Amostras de óleo de farelo de arroz & mg de $\gamma$-orizanol.kg ${ }^{-1}$ \\
\hline Bruto & 106,41 \\
Refinado em condições brandas & 97,95 \\
Comercial & 0,16 \\
\hline
\end{tabular}

${ }^{*}$ Média de duas repetições.

va-se que o óleo refinado em condições brandas possui maior resistência à oxidação, visto que a sua estabilidade oxidativa é 33\% maior que a do óleo comercial. Este comportamento é devido ao poder antioxidante do $\gamma$-orizanol, o qual foi preservado pelo refino físico em condições brandas.

Tabela 4. Período de indução do óleo de farelo de arroz obtido pelo refino físico em condições brandas e do óleo comercial a $110^{\circ} \mathrm{C}(5,0 \mathrm{~g}$ de amostra e 9,0 L.h.' de ar).

\begin{tabular}{ccc}
\hline Temperatura $\left({ }^{\circ} \mathrm{C}\right)$ & $\begin{array}{c}\text { Óleo refinado em } \\
\text { condições brandas }\end{array}$ & Óleo comercial \\
\hline $110^{\circ} \mathrm{C}$ & 13,3 horas & 8,9 horas \\
\hline
\end{tabular}

Na Tabela 5, são representadas as medidas de cor Lovibond obtidas para o óleo de farelo de arroz nas diferentes etapas do refino físico comparadas ao óleo comercial. Pelo fato de que se adotou neste trabalho o refino físico em condições brandas, a destruição térmica dos carotenóides foi minimizada, resultando em óleos desodorizados com teores mais altos de carotenóides, conhecidos como anticancerígenos. A técnica para viabilizar esta leitura mundialmente aceita é a determinada de cor Lovibond. Valores mais altos de leitura R (vermelho) e Y (amarelo) traduzem maiores concentrações de carotenóides. A aparência do óleo é a primeira barreira para aceitação do produto pelos consumidores $^{24}$. Nota-se que o óleo comercial possui maior intensidade de cor amarela em relação ao óleo obtido pelo refino físico sob condições brandas, que possui maior intensidade de cor vermelha. A retirada dos corantes dos óleos vegetais é mais uma questão de exigência dos consumidores, do que um requisito para sua boa qualidade. Na realidade, os corantes naturais nunca são prejudiciais, ao contrário, com sua retirada, o óleo pode até sofrer diminuição em suas propriedades alimentícias e nutritivas, pois é inevitável a perda associada com os corantes, de vitaminas e alguns fatores de crescimento $^{32}$.

Tabela 5. Medidas de Cor Lovibond em amostras de óleo de farelo de arroz.

\begin{tabular}{lc}
\hline \multicolumn{1}{c}{ Amostras } & Cor Lovibond (cubeta 5,1/4")* \\
\hline Óleo bruto & $54,0 \mathrm{Y} / 15,1 \mathrm{R} /(0 \mathrm{~B})$ \\
Óleo degomado & $34,0 \mathrm{Y} / 9,0 \mathrm{R} ;$ (0 B) \\
Óleo clarificado** & $50,5 \mathrm{Y} / 10,75 \mathrm{R}(0 \mathrm{~B})$ \\
Óleo desodorizado** & $35,7 \mathrm{Y} / 10,0 \mathrm{R} ;$ (0 B) \\
Óleo refinado winterizado** & $32,0 \mathrm{Y} / 7,0 \mathrm{R} ;(0 \mathrm{~B})$ \\
Óleo comercial*** & $70,0 \mathrm{Y} / 3,1 \mathrm{R} ;(0 \mathrm{~B})$ \\
\hline
\end{tabular}

${ }^{*}$ Média de três repetições; ${ }^{* *}$ Obtidos por refino físico em condições brandas; ${ }^{* * *}$ Obtido pelo refino químico.

\section{Conclusões}

A nova tecnologia de obtenção de óleo de farelo de arroz através de expeller, fornece óleo de baixa acidez quando 


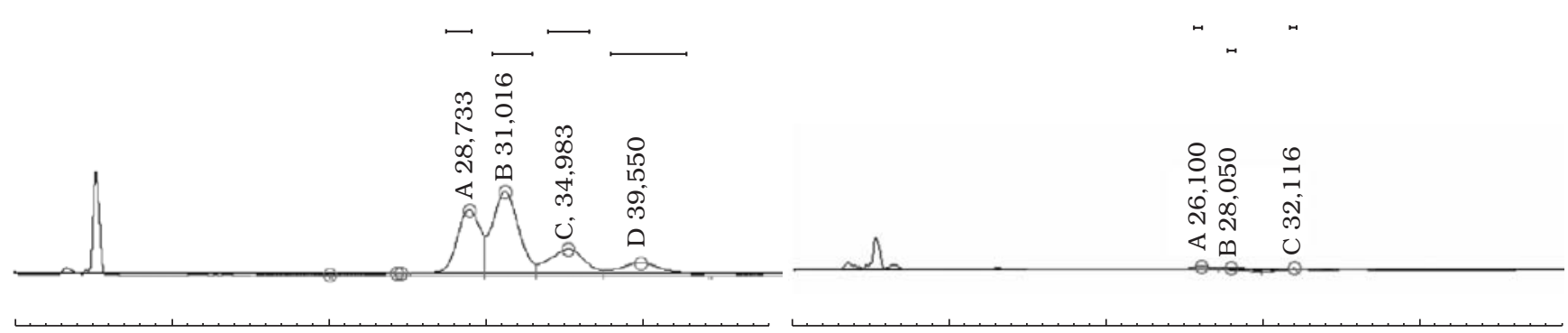

(c)

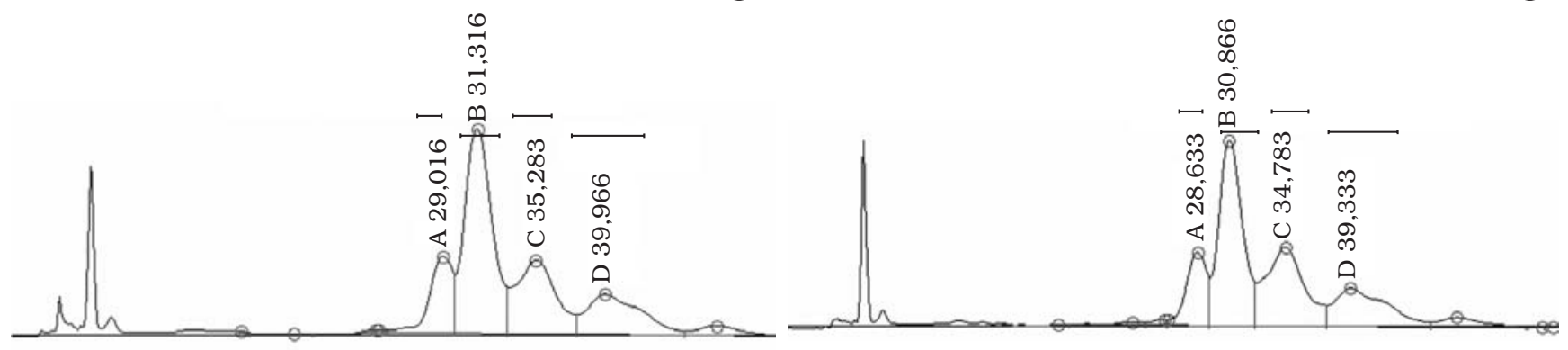

Figura 4. Cromatograma da composição de $\gamma$-orizanol, sendo: a) Padrão; b) Óleo comercial de farelo de arroz; c) Óleo bruto de farelo de arroz; e d) Óleo refinado de farelo de arroz (em condições brandas). Pico A = cicloartenil ferulato; B = 24-metileno cicloartenil ferulato; $\mathrm{C}=$ campesteril ferulato; e $\mathrm{D}=\beta$-sitosteril ferulato e cicloartenil ferulato.

proveniente de um farelo de arroz parboilizado. Pelo fato de não haver extração por solvente, sendo que o óleo apresenta também coloração mais clara e baixo teor de fósforo, estes parâmetros possibilitam o refino físico alternativo ao refino químico tradicional, que elimina todo o $\gamma$-orizanol do óleo ocluído no sabão.

O refino físico a baixa temperatura permite preservar para o consumidor os carotenóides, o teor inicial de $\gamma$-orizanol, proporcionando a este óleo uma excelente estabilidade oxidativa.

\section{Agradecimentos}

Ao Conselho Nacional de Desenvolvimento Científico e Tecnológico (CNPq), à Coordenação de Aperfeiçoamento de Pessoal de Nível Superior (CAPES), pela concessão das bolsas de mestrado e doutorado.

Aos técnicos do laboratório de Óleos e Gorduras pelo apoio na utilização dos equipamentos, em especial a Renato Grimaldi e Rosana Moreira.

À empresa Urbano Agroindústrias Ltda pela doação da amostra de óleo de farelo de arroz bruto.

\section{Referências bibliográficas}

1. ANVISA. Agência Nacional de Vigilância Sanitária. Resolução $\mathrm{n}^{\circ} 482$ de 23 de setembro de 1999. Regulamento Técnico para Fixação de Identidade e Gualidade de Óleos e Gorduras Vegetais. Diário Oficial da República Federativa do Brasil, Brasília, DF, 13 de outubro de 1999.

2. ANVISA. Agência Nacional de Vigilância Sanitária. Resolução $\mathrm{n}^{\circ} 270$ de 22 de setembro de 2005. Regulamento Técnico para Óleos Vegetais, Gorduras Vegetais e Creme Vegetal. Diário Oficial da República Federativa do Brasil, Brasília, DF, 23 de setembro de 2005.

3. AOCS. American Oil Chemists' Society. "Official Methods and Recommended Practices of the American Oil Chemists' Society", $5^{\circ}$ edition, Champaign, 2002.

4. BELLA CRUZ, A. Lipase de farelo de arroz: extração, imobilização e aplicação. Campinas, 2000, 105 p. Tese (Doutorado em Tecnologia de Alimentos) - Faculdade de Engenharia de Alimentos, Universidade Estadual de Campinas.

5. BERGER, A.; REIN, D.; SCHÄFER, A.; MONNARD, I.; GREMAUD, G.; LAMBELET, P.; BERTOLI, C. Similar cholesterol-lowering properties of rice bran oil, with varied $\gamma$-oryzanol, in mildly 
hypercholesterolemic men. European Journal of Nutrition, v. 44, n. 3, p. 1-11, 2004.

6. BHATTACHARYYA, A. C.; MAJUMDAR, S.; BHATTACHARYYA, D. K. Edible quality rice bran oil from high FFA rice bran oil by miscela refining. Journal of Amercian Oil Chemists' Society, v. 63, n. 9, p. 1189-1191, 1986.

7. BHATTACHARYYA, A. C.; MAJUNDAR, S.; BHATTACHARYYA, D. $\mathrm{K}$. Refining of high FFA rice bran oil by isopropanol extraction and alkali neutralization. Oleagineux, v. 42, n. 11, p. 431-433, 1987.

8. CODEX ALIMENTARIUS (FAO/WHO). Codex Standard for Named Vegetable Oils. Codex-Stan 210 (Amended 2003, 2005). p.1.13, Roma, 2005.

9. DE, B. K.; BHATTACHARYYA, D. K. Deacidification of high-acid bran oil by reesterification with monoglyceride. Journal of the American Oil Chemists' Society, v. 76, n. 10, p. 1243-1246, 1999.

10. DE, B. K.; DAS, R.; DUTTA, B. K.; BHATTACHARYYA, D. K. Membrane degguming and dewaxing of rice bran oil and its refining. Fett/Lipid, v. 100, n. 9, p. 416-421, 1998.

11. DORSA, R. Tecnologia de Óleos Vegetais. $1^{\circ}$ edição. Westaflia Separator do Brasil Ltda. Campinas, 2004.

12. ELMADFA, I. Physiologische beterung Unverseifbarer Komponenten von Nahrungsfetten. Fat Science Technology, v. 97 , n. 3, p. 85-90, 1995.

13. ESTEVES, W.; GONÇALVES, L. A. G.; BARRERA-ARELlANO, D. Metodologia padrão alemã para análise de gordura e outros lipídeos (Tradução de: Deutsche Einheitsmethodem zur Untersuchung von Fetten, Fettprodukten, Tensiden und verwandten Stoffen Abteilung A, B, C, F). Campinas. 1995.

14. FOOD AND AGRICULTURE ORGANIZATION OF THE UNITED NATIONS (FAO). http://faostat.fao.org/faostat/collections?subset =agriculture. Acesso em: 18 maio. 2006.

15. GINGRAS, L. Refining of rice bran oil. Inform, v. 11, n. 11, p. 1196-1203, 2000.

16. GONÇALVES, L. A. G., Comunicação Pessoal. 2006.

17. GOPALA KRISHNA, A. G.; KHATOON, S.; SHIELA, P. M.; SARMANDAL, C. V.; INDIRA, T. N.; MISHRA, A. Effect of refining of crude rice bran oil on the retention of oryzanol in the refined oil. Journal of the American Oil Chemists Society, v. 78, n. 2, p. 127-131, 2001.

18. GUPTA, H. P. Rice bran offers India an oil source. Journal of the American Oil Chemists' Society, v. 66, n. 5, p. 620-623, 1989.

19. HARTMAN, L.; LAGO, R.C.A. Rapid preparation of fatty acid methyl esters from lipids. Laboratory Practice, v. 22, n. 8, p. 475-476, 1973.

20. HEGSTED, M.; KOUSIK, C.S. Rice bran and rice bran oil may lower heart disease risk by decreasing cholesterol synthesis in the body. Louisiania Agriculture, v. 37, n. 2, p. 16-17, 1994.

21. HEMAVATHY, J.; PRABHAKAR, J. V. Lipid composition of rice (Oryza Sativa L.) bran. Journal of the American Oil Chemists' Society, v. 64, n. 7, p. 1016-1019, 1987.

22. JULIANO, C.; COSSU, M.; ALAMANNI, M. C.; PIU, L. Antioxidant activity of gamma-oryzanol: mechanism of action and its effect on oxidative stability of pharmaceutical oils. International Journal of Pharmaceutics, v. 299, n. 1-2, p. 146-154, 2005.

23. KAO, C.; LUH, B. S. Rice oil. In: LUH, B. S. Rice: utilization. $2^{\circ}$ ed. New York: Van Nostrand Reinhold, 1991, cap. 13, p. 295-311.
24. LAWSON, H. Food oils and fats: technology, utilization and nutrition. New York: Chapman \& Hall, 1995.

25. LUH, B.; BARBERS, S.; BARBER, C. B. Rice bran: chemistry and technology. In: LUH, B.S. Rice: utilization. 2 ed. New York: Van Nostrand Reinhold, 1991. cap. 14, p. 313-363.

26. MCCASKILL, D.R.; ZHANG, F. Use of rice bran oil in foods. Food Technology, v. 53, n. 2, p. 50-52, 1999.

27. NICOLOSI, J. R.; AUSMAN, L. M.; HEGSTED, D. M. Rice bran oil lowers serum total and low density lipoprotein cholesterol and apo B levels in nonhuman primates. Atherosclerosis, v. 88, n. 2-3, p. 133-142, 1991.

28. NORTON, R. A. Quantitation of steryl ferulate and p-coumarate esters from corn and rice. Lipids, v. 30, n. 3, p. 269-274, 1995.

29. ORTHOEFER, F. T. Rice bran oil: Healthy lipid source. Food Technology, v. 50, n.12, p. 62-64, 1996.

30. ORTHOEFER, F. T. Rice bran oil. In SHAHIDI, F. ( $6^{\circ}$ ed) Bailey's industrial oil \& fat products. New Jersey, John Wiley \& sons, 2005. v. 2, cap. 10, p. $465-489$.

31. ROGERS, E. J.; RICE, S. S. M.; NICOLOSI, R. J.; CARPENTER, D. R.; MCCLELLAND, C. A.; ROMANCZYK, L. J. Identification and quantification of $\gamma$ - orizanol components and simultaneous assessment of tocols in rice bran oil. Journal of the American Oil Chemists' Society, v. 70, n. 3, p.301-307, 1993.

32. ROHR, R. Óleos e gorduras vegetais e seus subprodutos protéicos. Campinas: UNICAMP, 1973.

33. SALUNKHE, D. K.; CHAVAN, J. K.; ADSULE, R. N.; KADAM, S. S. Rice. In: World oilseeds chemistry, technology, and utilization. 1 ed. New York: Van Nostrand Reinhold, 1991. cap. 12, p. 424-448.

34. SCAVARIELLO, E. M. S. Recuperação de $\gamma$ - orizanol da borra de neutralização de óleo de farelo de arroz. Campinas, 1997, 73 p.Tese (Mestrado em Tecnologia de Alimentos) - Faculdade de Engenharia de Alimentos, Universidade Estadual de Campinas.

35. SCAVARIELLO, E. M. S.; BARRERA-ARELLANO, D. Optimización del proceso de acidulación de la borra de neutralización de aceite de salvado de arroz. Grasas y Aceites, v. 55, n. 2, p. 155-159, 2004.

36. SEETHARAMAIAH, G. S.; CHANDRASEKHARA, N. Comparative hypocholesterolemic activities of oryzanol, curcumin and ferulic acids in rats. Journal of Food Science and Technology, v. 30, n. 4, p. 249-252, 1993.

37. SEETHARAMAIAH, G. S.; PRABAKAR, J. V. $\gamma$ - orizanol content of Indian rice bran oil and its extraction from soap stock. Journal of Food Science and Technology, v. 23, n. 5, p. 270-273, 1986.

38. SILVA, M. A.; SANCHES, C.; AMANTE, E. R. Prevention of hidrolytic rancidity in rice bran. Journal of Food Engineering, v. 75, n. 4, p. 487-491, 2006.

39. SUGANO, M.; TSUJI, E. Rice bran oil and cholesterol metabolism. The Journal of Nutrition. Present in a VII ${ }^{\text {th }}$ Asian conference of nutrition: Lipid symposium proceedings, p. 521S-524S, 1997.

40. SWERN, D. Bailey's Industrial Oil and Fat Products. $3^{\text {th }}$ ed. New York. John Wiley \& Sons. 1964.

41. TANAKA, A.; TANABE, K.; KATO, A.; MURAMATSU, J. Quantitative analysis of ferulates in rice bran oil by high performance liquid chromatography. Journal Japan Oil Chemists Society, v. 26, n. 2, p. 119-122, 1977. 
42. WANG, T.; HICKS, K. B.; MOREAU, R. Antioxidant activity of phytosterols, oryzanol, and other phytosterol conjugates. Journal of the American Oil Chemists' Society, v. 79, n. 12, p. 1201-1206, 2002.

43. YOKOCHI, K. Rice bran processing for the production of rice bran oil and characteristics and uses of the oil and deoiled bran. In:
Rice BY - PRODUCTS CONFERENCE, 1974, Valencia, Espanha, p. 2-37.

44. YOON, S. H.; KIM, S. K. Oxidative stability of high-fatty acid rice bran oil at different stages of refining. Journal of the American Oil Chemists' Society, v. 71, n. 2, p. 227-229, 1994. 